\title{
Analysis of the 19th Century Historic Archaeological Material Culture Remains from the Browning Site in Smith County, Texas
}

Timothy K. Perttula

Heritage Research Center, Stephen F. Austin State University

Mark Walters

Heritage Research Center, Stephen F. Austin State University

Follow this and additional works at: https://scholarworks.sfasu.edu/ita

Part of the American Material Culture Commons, Archaeological Anthropology Commons, Environmental Studies Commons, Other American Studies Commons, Other Arts and Humanities Commons, Other History of Art, Architecture, and Archaeology Commons, and the United States History Commons

Tell us how this article helped you.

This Article is brought to you for free and open access by the Center for Regional Heritage Research at SFA ScholarWorks. It has been accepted for inclusion in Index of Texas Archaeology: Open Access Gray Literature from the Lone Star State by an authorized editor of SFA ScholarWorks. For more information, please contact cdsscholarworks@sfasu.edu. 


\section{Analysis of the 19th Century Historic Archaeological Material Culture Remains}

from the Browning Site in Smith County, Texas

\section{Creative Commons License}

\section{(c) (1) (8)}

This work is licensed under a Creative Commons Attribution-NonCommercial 4.0 International License 


\title{
Analysis of the 19th Century Historic Archaeological Material Culture Remains from the Browning Site in Smith County, Texas
}

\author{
Timothy K. Perttula and Mark Walters
}

\section{SITE SETTING AND EXCAVATIONS}

The Browning site (41SM195A) is located on a $3800 \mathrm{~m}^{2}$ alluvial terrace that overlooks the Auburn Creek floodplain in eastern Smith County, Tcxas. This setting is near the headwaters of a stream system in the Harris Creek drainage; Harris Creek meets the Sabine River ca. $34 \mathrm{~km}$ to the north. In the vicinity of the Browning site, the valley, being narrow with stcep valley walls, offers few locations suitable for either prehistoric or historic occupations. Soils here are Entisols; they vary in depth from $30-70 \mathrm{~cm}$ across the landform, terminating at a sandstone C-horizon. These are soils that formed mostly under forest vegetation and are dominantly sandy or loamy (Hatherly 1993). The Browning site falls within the Pineywoods vegetation area and represents the western extent of the pine and deciduous forests of the Southeastern U.S. coastal plain (Diggs et al. 2006).

Archaeological investigations at the Browning site have been carried out intermittingly for several years by the junior author (Figure 1). That work has led to the recognition that it is a stratified site with two very distinct occupations, an early to mid-19th ccntury assemblage of historic artifacts primarily in an upper zone $(0-20 \mathrm{~cm}$ bs) overlying a buried (20-50 cm bs) Late Woodland period occupation (Walters 2004a, 2004b, 2009; Shafer and Walters 2010). The historic occupation is in the center of the terrace, covering approximately $500 \mathrm{~m}^{2}$. The historic artifacts are found primarily in the upper sediments, but due probably to pedoturbations, they have been found as deep as $50 \mathrm{~cm}$ in the underlying prehistoric archacological deposits.

Excavations at the Browning site have consisted of 41 I I m units (with a total excavated volume of 20.4 $\mathrm{m}^{3}$ ) and 22 shovel tests (sec Figure 1). Surface collections were obtained from the site in 1996 and 2002.

The 22 shovel tests excavated at the site were conducted first to better ascertain the limits of the site and identify areas of concentrated cultural activity; ST 4, 8-9, 12, and 19 contained 19th century historic artifacts (see Figure 1). Once a buried prehistoric occupation zone was identified, units were placed primarily in cardinal directions to better define the occupation zone's boundaries and levels of occupational intensity, and also sample the overlying 19th century component. The $1 \times 1 \mathrm{~m}$ units were excavated in arbitrary $10 \mathrm{~cm}$ levels and the soil was dry-screened for artifacts through 1/4-inch hardware cloth except for a fine screen sample from Unit 1 that was water-screened through $1 / 32$-inch mesh. A level sheet was completed at the end of cach Icvel. Profiles were drawn of one wall of each unit or a common wall when several units were joined. The shovel tests followed the same procedures, except they were excavated in arbitrary $20 \mathrm{~cm}$ levels.

One fcaturc (Fcature 2) had four relined earthenware sherds (as well as two prehistoric artifacts) and 15 small picces of animal bone. This was a shallow pit with a very dark grayish-brown sandy loam fill (with charcoal flecks) and a rounded bottom that was $63 \mathrm{~cm}$ in diameter and extended from $22-29 \mathrm{~cm}$ bs.

\section{TH CENTURY HISTORIC ARTIFACTS FROM THE BROWNING SITE}

The excavations at the Browning site have rccovercd a total of 36019 th century artifacts from surface collections $(n=17)$, shovel testing $(n=7,1.4$ artifacts per positive shovel test, or ca. 11.2 artifacts per $\mathrm{m}^{2}$ ), and the $1 \times 1 \mathrm{~m}$ unit excavations ( $\mathrm{n}=336$, or 8.2 artifacts per $\mathrm{m}^{2}$ ) (Table 1). Most of the recovered historic artifacts are sherds from refined earthenware (i.c., whiteware and porcelain) plates and cups $(81.3 \%)$, followed hy artifacts of iron (mainly cut nails) $(11.4 \%)$, stoneware, stoneware pipes and yellowware sherds $(3.9 \%)$, and bottle glass $(3.6 \%)$. 


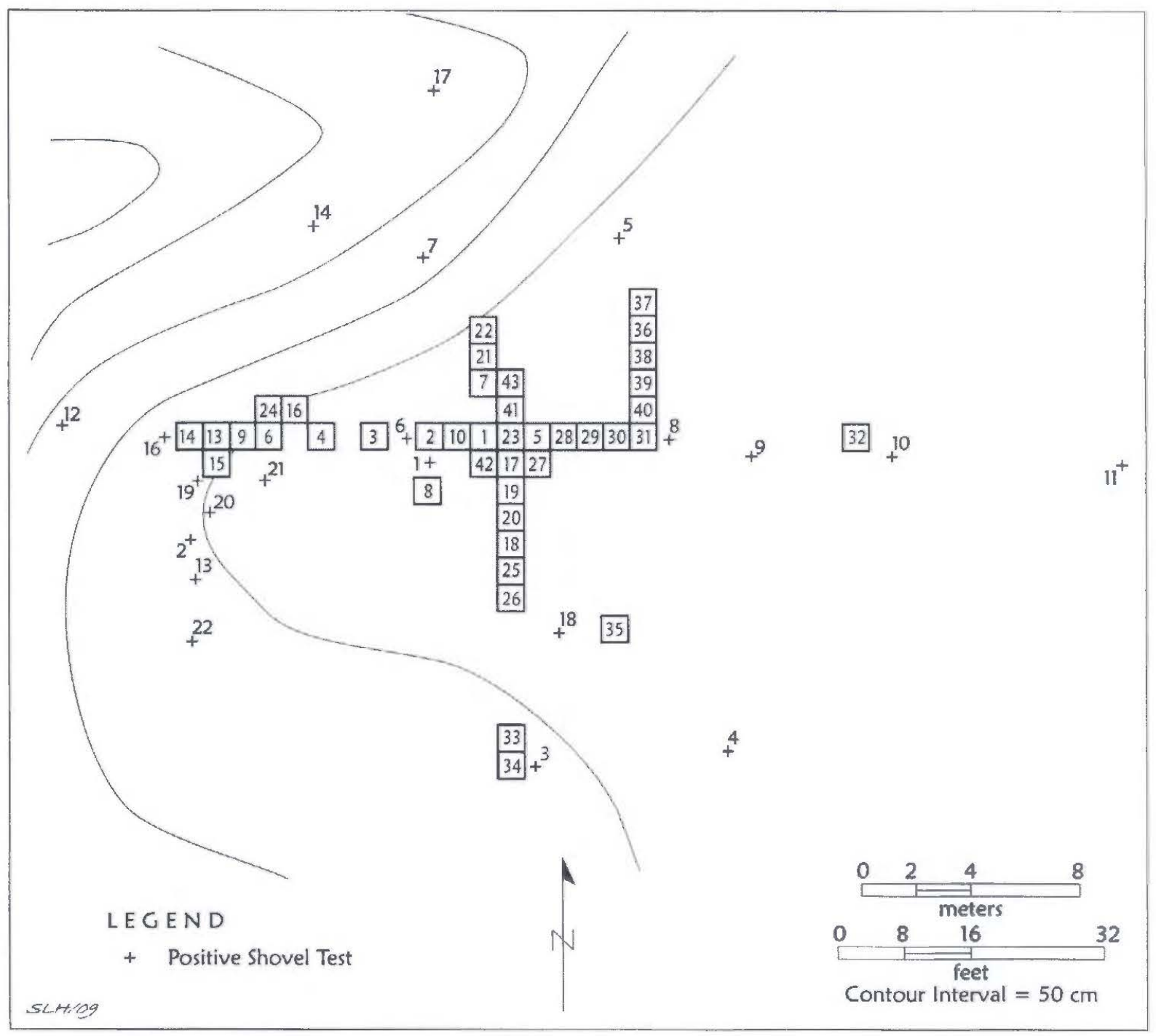

Figure 1. Map of the Browning site archaeological investigations.

By depth, 86\% of the 19th century artifacts from the shovel tests were found between $0-20 \mathrm{~cm}$ bs, with one artifact recovered from $20-40 \mathrm{~cm}$ bs. In the hand-excavated units, the preponderance of the historic artifacts is also from $0-20 \mathrm{~cm}$ bs $(64.2 \%)$, but there are significant amounts of historic artifacts in the $20-30 \mathrm{~cm}(24.8 \%), 30-40 \mathrm{~cm}(8.3 \%)$, and $40-50 \mathrm{~cm}$ bs (1.5\%) levels; $1.2 \%$ of the historic artifacts from the Browning site are from Feature 2 (22-29 cm bs).

The spatial density of 19 th century artifacts from the site indicates that there are three small clusters of units that have higher densities of artifacts in the excavation area (Figure 2). The first is at the western edge of the terrace, in Units 6, 13-15, and 24 (12-18 artifacts per $\left.\mathrm{m}^{2}\right)$; the second cluster is in the central part of the terrace (Units 17, 21, 22, 28-31, 40 , and 42 , with $9-13$ artifacts per $\mathrm{m}^{2}$ ); and a third clustcr at the southern edge of the terrace (Units 33$34,14-21$ artifacts per $\mathrm{m}^{2}$ ). At the present time, it is not known if these higher density spatial clusters of artifacts correspond to functionally different parts of the 19th century occupation, or if they simply represent discrete trash disposal areas.

Refined earthenwares-including whiteware and porcelain - are abundant in the Browning site 19th century artifact assemblage (Table 2). The whitewares, both plain and decorated sherds (about $22 \%$ of the whiteware sherds have a decorative element) from vessels that were likely made by English potteries beginning in the 1830s (Majewski and O'Brien 1987), account for about $98 \%$ of the refined 


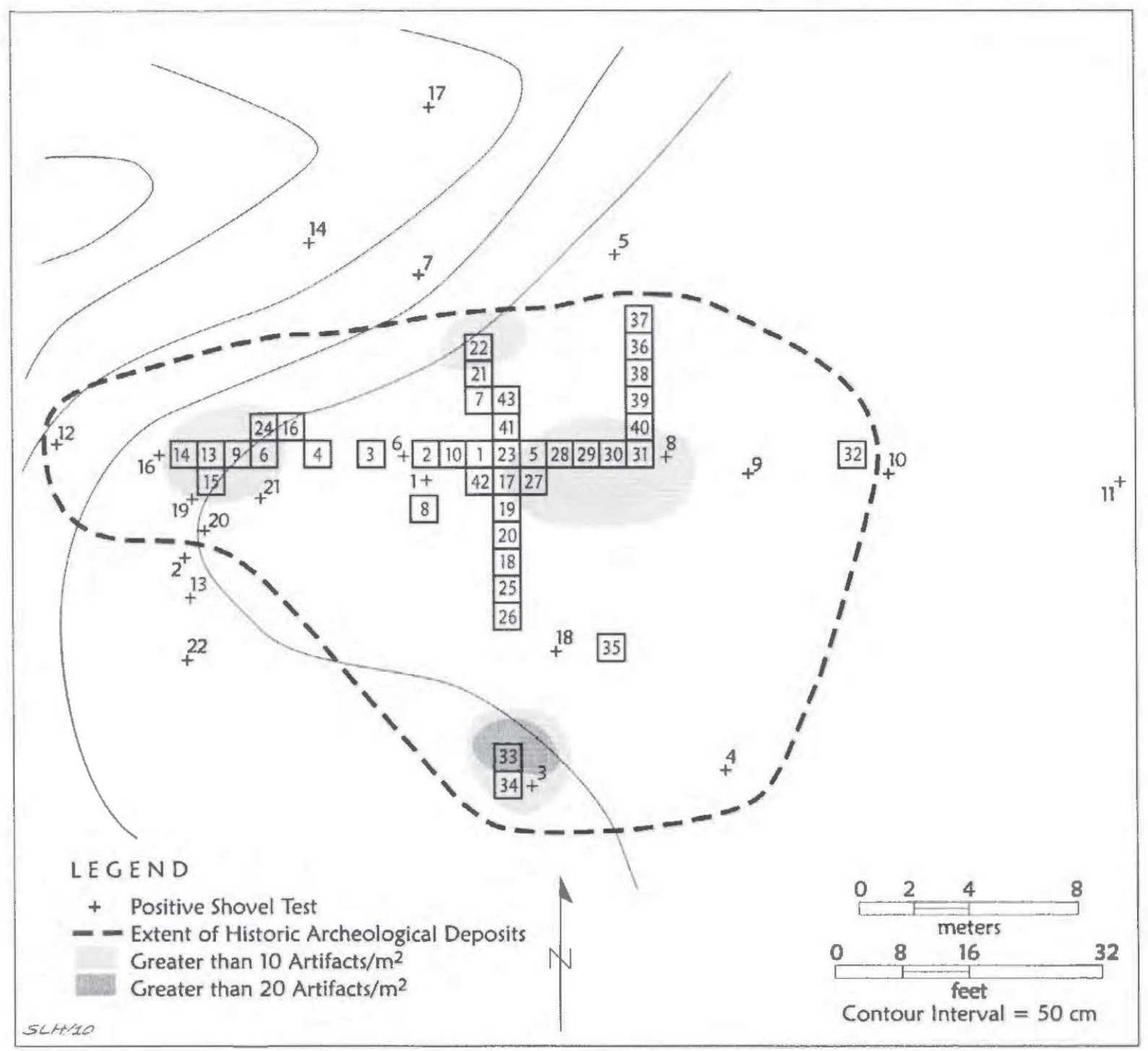

Figure 2. The distribution of historic 19 th century artifacts from the Browning site.

earthenwares, and the remaining $2 \%$ are sherds from porcelain or bone-china vessels.

The decorated whitewares are divided into eight different categories based on the kind of decoration (i.e., hand-painted, transfer-printed, annular ware, shell-edged, etc.) found on individual sherds in the assemblage (Table 3). Sherds from annular ware vessels are the most common, comprising $39 \%$ of the decorated sherds, followed by hand-painted sherds $(28 \%)$, blue shell-edged sherds $(23 \%)$, and transfer-printed $(4.7 \%)$. Sponge ware and flown blue sherds account for the remaining $4.7 \%$ of the decorated sherds from the Browning site.

In general, the decorated whiteware from the Browning site would seem to have been made and used between the 1830s and 1860 (cf. Price 1979;
Majewski and O'Brien 1984, 1987). All of these decorated ceramic sherds are from vessels probably of English manufacture, and they likely were obtained through shipments of goods from New Orleans brought up the Red River and Caddo Lake to Jefferson, and then carricd overland to distributors and stores.

Annular wares (Figure 3) at the site have earthy color tones. These include brown, black, and yellow bands of varying widths as well as gray, yellow, white, and blue zones, as well as cat's eye dots (Majewski and O'Brien 1987:163), suggesting these sherds are from early (ca. 1840s) annular ware.

The hand-painted sherds from the site are from cups primarily decorated with fine line polychrome and monochrome floral motils (including petals and 
Table 1. Historic Artifacts from the Browning site.

\begin{tabular}{|c|c|c|c|c|}
\hline Provenience & Mélal & $\begin{array}{l}\text { Bottle } \\
\text { Glass }\end{array}$ & $\begin{array}{l}\text { Refined } \\
\text { Earthenware }\end{array}$ & Stoneware \\
\hline Surfati & - & & 17 & - \\
\hline $\mathrm{ST} 4$ & - & 1 & - & - \\
\hline ST \& & & 1 & 1 & \\
\hline ST 9 & - & - & 1 & - \\
\hline ST 12 & - & & 1 & 1 \\
\hline ST 19 & - & & - & 1 \\
\hline Unit 1 & & - & $x$ & \\
\hline Unit 2 & - & - & 3 & - \\
\hline Unit 3 & - & - & 3 & - \\
\hline Unit 4 & - & - & 3 & - \\
\hline Unit 5 & 1 & - & 3 & - \\
\hline Unit 6 & 2 & 1 & $x$ & 1 \\
\hline Unit 7 & & - & 5 & - \\
\hline Unit 8 & - & - & 2 & 1 \\
\hline Unit 9 & - & & 7 & - \\
\hline Unit 10 & - & - & 5 & \\
\hline Unit 13 & 3 & 2 & 13 & - \\
\hline Unit 14 & - & 1 & 14 & - \\
\hline Unit 15 & 1 & 4 & 9 & 2 \\
\hline Unit 16 & - & - & 7 & 2 \\
\hline Unit 17 & 1 & - & 12 & - \\
\hline Unit 18 & - & & 7 & - \\
\hline Unit 19 & 1 & & 3 & - \\
\hline Unil 20 & 2 & - & 4 & \\
\hline Unit 21 & 2 & - & 7 & - \\
\hline Unit $22 * *$ & 1 & - & 10 & - \\
\hline Unit 23 & - & 1 & 6 & - \\
\hline Unit 24 & 2 & - & 11 & 1 \\
\hline Unit 25 & - & - & 1 & - \\
\hline Unit 26 & - & & 3 & - \\
\hline Unit 27 & - & - & 4 & \\
\hline Unit 28 & 1 & - & 10 & - \\
\hline Unit 29 & - & - & 11 & 1 \\
\hline Unit 30) & 1 & - & 11 & 1 \\
\hline Unit 31 & 2 & & 9 & 1 \\
\hline Unit 32 & & - & 2 & 2 \\
\hline Unit 33 & 2 & - & 19 & - \\
\hline Unit 34 & 6 & 1 & 7 & - \\
\hline Unit 35 & 2 & - & 6 & - \\
\hline Unit 36 & $2+$ & & 4 & - \\
\hline Unit 37 & 3 & & 1 & \\
\hline
\end{tabular}


Table 1. Historic Artifacts from the Browning site, cont.

\begin{tabular}{|c|c|c|c|c|}
\hline Provenience & Metal & $\begin{array}{l}\text { Bottle } \\
\text { Glass }\end{array}$ & $\begin{array}{c}\text { Refined } \\
\text { Earthenware }\end{array}$ & Stoneware* \\
\hline Unit 38 & 2 & - & 5 & - \\
\hline Unit 39 & 1 & - & 5 & - \\
\hline Unit 40 & - & - & 9 & - \\
\hline Unit 41 & 1 & 1 & 3 & - \\
\hline Unit 42 & 1 & - & 8 & - \\
\hline Unit 43 & 1 & - & 4 & - \\
\hline Totals & 41 & 13 & 292 & 14 \\
\hline \multicolumn{5}{|c|}{$\begin{array}{l}\text { *including pipe sherds and yellowware sherds; ** includes for } \\
\text { ( } 22-29 \mathrm{~cm} \text { bs) } \\
\text { +late } 19 \text { th-early } 20 \text { th century shotgun shell not included in the }\end{array}$} \\
\hline $\begin{array}{l}\text { Provenience } \\
(\mathrm{cm} \mathrm{bs})\end{array}$ & $\begin{array}{c}\text { Plain } \\
\text { Whiteware }\end{array}$ & $\begin{array}{l}\text { Decorated } \\
\text { Whiteware }\end{array}$ & $\begin{array}{c}\text { Plain } \\
\text { Porcelain }\end{array}$ & $\mathrm{N}$ \\
\hline Surface & 14 & 2 & 1 & 17 \\
\hline ST $8,20-40$ & 1 & - & - & 1 \\
\hline ST $9,0-20$ & 1 & - & - & 1 \\
\hline ST $12,0-20$ & 1 & - & - & 1 \\
\hline Unit $1,0-10$ & 1 & 1 & - & 2 \\
\hline Unit $1,10-20$ & $5^{*}$ & - & 1 & 6 \\
\hline Unit $2,0-10$ & 2 & - & - & 2 \\
\hline Unit $2,40-50$ & 1 & - & - & 1 \\
\hline Unit $3,0-10$ & 1 & 1 & - & 2 \\
\hline Unit $3,10-20$ & 1 & - & - & 1 \\
\hline Unit $4,0-10$ & 2 & 1 & - & 3 \\
\hline Unit $5,0-10$ & 1 & - & - & 1 \\
\hline Unit $5,10-20$ & 1 & 1 & - & 2 \\
\hline Unit $6,10-20$ & 6 & 1 & - & 7 \\
\hline Unit $6,20-30$ & 1 & - & - & 1 \\
\hline Unit $7,0-10$ & - & 3 & - & 3 \\
\hline Unit $7,10-20$ & - & 1 & 1 & 2 \\
\hline Unit $8,0-10$ & 2 & - & - & 2 \\
\hline
\end{tabular}


18 Journal of Northeast Texas Archaeology 34 (2011)

Table 2. Refined Earthenwares from the Browning site, cont.

\begin{tabular}{|c|c|c|c|c|}
\hline $\begin{array}{l}\text { Provenience } \\
\text { (cm bs) }\end{array}$ & $\begin{array}{c}\text { Plain } \\
\text { Whiteware }\end{array}$ & $\begin{array}{l}\text { Decorated } \\
\text { Whiteware }\end{array}$ & $\begin{array}{c}\text { Plain } \\
\text { Porcelain }\end{array}$ & $\mathrm{N}$ \\
\hline Unit $9,0-10$ & 1 & - & - & 1 \\
\hline Unit $9,10-20$ & 3 & 2 & - & 5 \\
\hline Unit $9,20-30$ & 1 & - & - & 1 \\
\hline Unit $10,0-10$ & 2 & - & - & 2 \\
\hline Unit $10,10-20$ & 2 & - & - & 2 \\
\hline Unit $10,20-30$ & 1 & - & - & 1 \\
\hline Unit $13,0-10$ & 2 & 1 & - & 3 \\
\hline Unit $13,10-20$ & 4 & 3 & - & 7 \\
\hline Unit $13,20-30$ & 3 & - & - & 3 \\
\hline Unit $14,0-10$ & 5 & 1 & - & 6 \\
\hline Unit $14,10-20$ & 4 & - & - & 4 \\
\hline Unit $14,20-25$ & 4 & - & - & 4 \\
\hline Unit $15,0-10$ & - & 1 & - & 1 \\
\hline Unit $15,10-20$ & 5 & 3 & - & 8 \\
\hline Unit $16,0-10$ & 3 & 2 & - & 5 \\
\hline Unit $16,10-20$ & - & 1 & - & 1 \\
\hline Unit $16,20-30$ & 1 & - & - & 1 \\
\hline Unit $17,0-10$ & 6 & 1 & - & 3 \\
\hline Unit $17,10-20$ & 2 & - & - & 2 \\
\hline Unit $17,20-30$ & 3 & - & - & 3 \\
\hline Unit $18,10-20$ & 6 & - & - & 6 \\
\hline Unit $18,20-30$ & 1 & - & - & 1 \\
\hline Unit $19,20-30$ & - & 2 & - & 2 \\
\hline Unit $19,40-50$ & 1 & - & - & 1 \\
\hline Unit $20,10-20$ & - & 1 & - & I \\
\hline Unit $20,20-30$ & 2 & 1 & - & 3 \\
\hline Unit $21,10-20$ & 1 & - & 1 & 2 \\
\hline Unit $21,20-30$ & 1 & 1 & - & 2 \\
\hline Unit $21,30-40$ & 2 & 1 & - & 3 \\
\hline Unit $22,0-10$ & 1 & - & - & 1 \\
\hline Unit $22,10-20$ & 3 & - & - & 3 \\
\hline Unit $22,20-30$ & 2 & - & - & 2 \\
\hline Unit 22, F. 2 & 4 & - & - & 4 \\
\hline
\end{tabular}


Table 2. Refined Earthenwares from the Browning site, cont.

\begin{tabular}{|c|c|c|c|c|}
\hline $\begin{array}{l}\text { Provenience } \\
\text { (cm bs) }\end{array}$ & $\begin{array}{c}\text { Plain } \\
\text { Whiteware }\end{array}$ & $\begin{array}{l}\text { Decorated } \\
\text { Whiteware }\end{array}$ & $\begin{array}{c}\text { Plain } \\
\text { Porcelain }\end{array}$ & $\mathrm{N}$ \\
\hline Unit 23, 10-20 & 5 & 1 & - & 6 \\
\hline Unit $24,0-10$ & 2 & 2 & - & 4 \\
\hline Unit $24,10-20$ & 5 & 2 & - & 7 \\
\hline Unit $25,10-20$ & 1 & - & - & 1 \\
\hline Unit $26,10-20$ & 2 & - & - & 2 \\
\hline Unit $26,20-30$ & 1 & - & - & 1 \\
\hline Unit $27,10-20$ & 2 & - & - & 2 \\
\hline Unit $27,20-30$ & $1^{*}$ & 1 & - & 2 \\
\hline Unit $28,10-20$ & $4 *$ & 1 & & 5 \\
\hline Unit $28,30-40$ & 3 & 1 & - & 4 \\
\hline Unit $28,40-50$ & 1 & - & - & 1 \\
\hline Unit $29,10-20$ & 4 & - & - & 4 \\
\hline Unit $29,20-30$ & 6 & - & - & 6 \\
\hline Unit $29,30-40$ & 1 & - & - & 1 \\
\hline Unit $30,0-10$ & 1 & - & - & 1 \\
\hline Unit $30,10-20$ & 1 & - & - & 1 \\
\hline Unit $30,20-30$ & 6 & 1 & - & 7 \\
\hline Unit $30,30-40$ & - & 1 & - & 1 \\
\hline Unit $30,40-50$ & 1 & - & - & 1 \\
\hline Unit $31,0-10$ & 3 & - & - & 3 \\
\hline Unit $31,10-20$ & 1 & - & - & 1 \\
\hline Unit $31,20-30$ & 1 & 2 & - & 3 \\
\hline Unit $31,30-40$ & - & 1 & - & 1 \\
\hline Unit $31,40-50$ & 1 & - & - & 1 \\
\hline Unit $32,0-10$ & 1 & - & - & 1 \\
\hline Unit $32,20-30$ & 1 & - & - & 1 \\
\hline Unit 33, 0-10 & 1 & 1 & - & 2 \\
\hline Unit $33,10-20$ & 1 & 1 & - & 2 \\
\hline Unit $33,20-30$ & 3 & 2 & - & 5 \\
\hline Unit $33,30-40$ & 10 & - & - & 10 \\
\hline Unit $34,10-20$ & - & - & 1 & 1 \\
\hline Unit $34,20-30$ & 4 & - & - & 4 \\
\hline Unit $34,30-40$ & 2 & - & - & 2 \\
\hline
\end{tabular}


Table 2. Refined Earthenwares from the Browning site, cont.

\begin{tabular}{|c|c|c|c|c|}
\hline $\begin{array}{l}\text { Provenience } \\
(\mathrm{cm} \mathrm{bs})\end{array}$ & $\begin{array}{c}\text { Plain } \\
\text { Whiteware }\end{array}$ & $\begin{array}{l}\text { Decorated } \\
\text { Whiteware }\end{array}$ & $\begin{array}{c}\text { Plain } \\
\text { Porcelain }\end{array}$ & $\mathrm{N}$ \\
\hline Unit $35,0-10$ & 3 & 2 & - & 5 \\
\hline Unit $35,30-40$ & 1 & - & - & 1 \\
\hline Unit $36,10-20$ & 1 & 1 & - & 2 \\
\hline Unit $36,20-30$ & - & 2 & - & 2 \\
\hline Unit $37,10-20$ & 1 & - & - & 1 \\
\hline Unit $38,10-20$ & 2 & 1 & - & 3 \\
\hline Unit $38,20-30$ & 1 & - & - & 1 \\
\hline Unit $38,30-40$ & 1 & - & - & 1 \\
\hline Unit $39,10-20$ & 2 & - & - & 2 \\
\hline Unit $39,20-30$ & 2 & 1 & - & 3 \\
\hline Unit $40,10-20$ & 1 & 2 & 1 & 4 \\
\hline Unit $40,20-30$ & 3 & 1 & - & 4 \\
\hline Unit $40,30-40$ & 1 & - & - & 1 \\
\hline Unit $41,0-10$ & - & 1 & - & 1 \\
\hline Unit $41,10-20$ & $2 *$ & - & - & 2 \\
\hline Unit $42,0-10$ & 2 & 2 & - & 4 \\
\hline Unit $42,10-20$ & $1^{*}$ & 1 & - & 2 \\
\hline Unit $42,20-30$ & 2 & - & - & 2 \\
\hline Unit $43,0-10$ & 1 & - & - & 1 \\
\hline Unit $43,10-20$ & 2 & 1 & - & 3 \\
\hline Totals & 222 & 64 & 6 & 292 \\
\hline
\end{tabular}

*includes plain rims $(\mathrm{n}=5)$ with non-scalloped lips and impressed lines

Table 3. Decorated Whiteware Sherds.

\begin{tabular}{lcccccccc}
\hline $\begin{array}{l}\text { Provenience } \\
(\mathrm{cm} \text { bs })\end{array}$ & HP-P & HP-FL & AW-CE & AW-B & TP-F & BSE & SP & FB \\
\hline Surface & - & - & - & - & - & $2 *$ & - & - \\
Unit 1, 0-10 & - & - & - & - & 1 & - & - & - \\
Unit 3, 0-10 & - & - & - & - & - & $1 *$ & - & - \\
Unit 4, 0-10 & - & 1 & - & - & - & - & - & -
\end{tabular}


Table 3. Decorated Whiteware Sherds, cont.

\begin{tabular}{|c|c|c|c|c|c|c|c|c|}
\hline $\begin{array}{l}\text { Provenience } \\
\text { (cm bs) }\end{array}$ & HP-P & HP-FL & AW-CE & AW-B & TP-F & BSE & SP & FB \\
\hline Unit $5,10-20$ & - & - & - & 1 & - & - & - & - \\
\hline Unit $6,10-20$ & - & 1 & - & - & - & - & - & - \\
\hline Unit 7, 0-10 & - & - & - & 3 & - & - & - & - \\
\hline Unit $7,10-20$ & - & - & - & - & $\begin{array}{c}1 \\
\text { (blue) }\end{array}$ & - & - & - \\
\hline Unit $9,10-20$ & 1 & - & 1 & - & - & - & - & - \\
\hline Unit $13,0-10$ & - & 1 & - & - & - & - & - & - \\
\hline Unit $13,10-20$ & - & 2 & - & - & - & - & - & 1 \\
\hline Unit $14,0-10$ & - & - & - & 1 & - & - & - & - \\
\hline Unit $15,0-10$ & - & 1 & - & - & - & - & - & - \\
\hline Unit $15,10-20$ & - & 3 & - & - & - & - & - & - \\
\hline Unit $16,0-10$ & - & - & - & - & - & $2 / 1 *$ & - & - \\
\hline Unit $16,10-20$ & - & - & - & - & - & - & 1 & - \\
\hline Unit $17,0-10$ & - & - & - & - & $\begin{array}{c}1+ \\
\text { (blue) }\end{array}$ & & & \\
\hline Unit $19,20-30$ & - & - & - & 1 & - & $1^{*}$ & - & - \\
\hline Unit $20,10-20$ & - & - & - & 1 & - & - & - & - \\
\hline Unit $20,20-30$ & - & - & - & 1 & - & - & - & - \\
\hline Unit $21,20-30$ & - & 1 & - & - & - & - & - & - \\
\hline Unit $21,30-40$ & - & - & - & 1 & - & - & - & - \\
\hline Unit $23,10-20$ & - & - & - & - & - & $1^{*}$ & - & - \\
\hline Unit $24,0-10$ & - & 2 & - & - & - & - & - & - \\
\hline Unit $24,10-20$ & - & 1 & - & 1 & - & - & - & - \\
\hline Unit $27,20-30$ & - & - & - & 1 & - & - & - & - \\
\hline Unit $28,10-20$ & - & - & - & 1 & - & - & - & - \\
\hline Unit $28,30-40$ & - & 1 & - & - & - & - & - & - \\
\hline Unit $30,20-30$ & - & - & - & 1 & - & - & - & - \\
\hline Unit $30,30-40$ & - & - & - & 1 & - & - & - & - \\
\hline Unit $31,20-30$ & - & 1 & - & 1 & - & - & - & - \\
\hline Unit $31,30-40$ & - & - & - & 1 & - & - & - & - \\
\hline Unit $33,0-10$ & - & - & - & 1 & - & - & - & - \\
\hline Unit $33,10-20$ & - & - & - & - & - & 1 & - & - \\
\hline Unit $33,20-30$ & - & - & - & 1 & - & $1^{*}$ & - & - \\
\hline Unit $35,0-10$ & - & - & - & 1 & - & 1 & - & - \\
\hline Unit $36,10-20$ & - & - & - & - & - & $1^{*}$ & - & - \\
\hline Unit $36,20-30$ & - & - & - & 2 & - & - & - & - \\
\hline Unit $38,10-20$ & - & - & - & - & - & $1^{*}$ & - & - \\
\hline Unit $39,20-30$ & - & - & - & - & - & $1^{*}$ & - & - \\
\hline Unit $40,10-20$ & - & 1 & - & - & - & $1^{*}$ & - & - \\
\hline Unit $40,20-30$ & - & - & - & - & - & - & 1 & - \\
\hline Unit $41,0-10$ & - & - & - & 1 & - & - & - & - \\
\hline
\end{tabular}


Table 3. Decorated Whiteware Sherds, cont.

\begin{tabular}{lcccccccc}
\hline $\begin{array}{l}\text { Provenience } \\
\text { (cm bs) }\end{array}$ & HP-P & HP-FL & AW-CE & AW-B & TP-F & BSE & SP & FB \\
\hline Unit 42, 0-10 & - & - & - & 1 & - & $1 *$ & - & - \\
Unit 42, 10-20 & - & - & - & 1 & - & - & - & - \\
Unit 43, 10-20 & 1 & - & - & - & - & - & - & - \\
\hline Totals & 2 & 16 & 1 & 24 & 3 & 15 & 2 & 1 \\
\hline
\end{tabular}

HP-P=hand-painted, polychrome; HP-fine-line and/or monochrome; AW-CE=annular ware, cat's eye; AW-B, annular ware, banded in brown, black, and yellow and with gray, yellow, and blue zones; TP-F=transfer-printed-floral; $\mathrm{BSE}=$ blue shell-edged; $\mathrm{SP}=$ sponged; $\mathrm{FB}=$ flown blue

*non-scalloped lip with impressed lines on the rim toriental motif

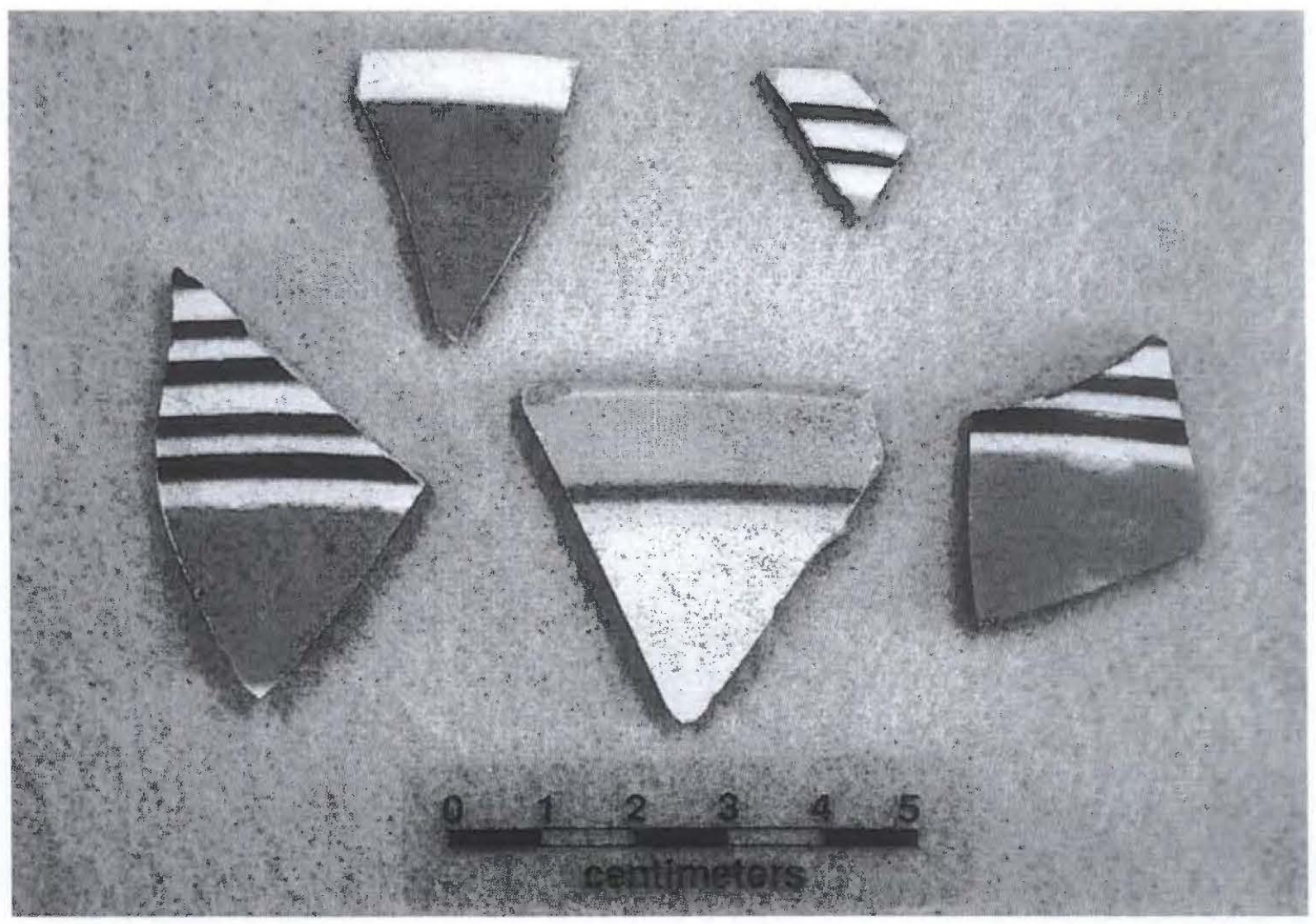

Figure 3. Annular wares with earth-toned bands and zones. Provenience: top row, left to right: Unit 28, 10-20 cm; Unit $31,20-30 \mathrm{~cm}$; bottom row, left to right: Unit 42, 10-20 cm; Unit 8, 0-10 cm; Unit 33, 0-10 cm.

branches) (Figure 4). The rim sherds have blue or black hand-painted rim lines on both the exterior and interior cup surfaces. These decorative elements are consistent with ca. 1840-1860 hand-painted whitcwares (Majewski and O'Brien 1987:157).
The shell-edged whiteware from the Browning site has a blue painted edge, impressed lines, and a non-scalloped lip (Figure 5); on three sherds, the lip form could not be determined because of breakage of the sherd. The absence of green shell-edged 


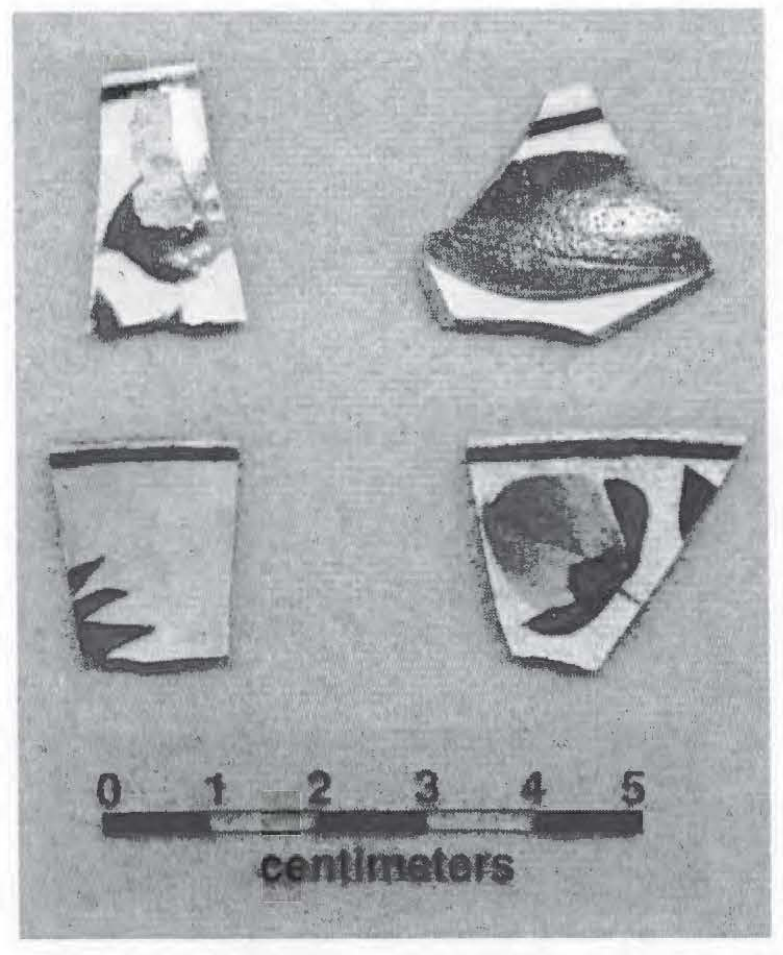

Figure 4. Hand-painted rim sherds. Provenience: top row, left to right: Unit $15,10-20 \mathrm{~cm}$; Unit $9,10-20 \mathrm{~cm}$; bottom row, left to right: Unit $6,10-20 \mathrm{~cm}$; Unit $24,0-10 \mathrm{~cm}$.

refined earthenware from the site is notable because green shell-edged plates began to diminish in frequency after 1800 relative to blue shell-edged plates (Sussman 2000:51).

The rim form of shell-edged ceramics is chronologically sensitive (cf. Hunter and Miller 1994, 2009). Plates and platters with unscalloped rims and impressed lines, like all the blue shell-edged whiteware from the Browning site, were being made by the 1840 s, while the earlier symmetrical scalloped shell-edged ware continued to be made into the 1830 s (Hunter and Miller 2009:13); this earlier form is absent in the Browning site artifact sample. There are five plain rims with unscalloped edges and impressed lines (see Table 2) that were likely made at the same time.

All three of the transfer-printed sherds have medium blue colors, and either floral (Figure 6, right) or oriental (Figure 6, left) motifs. The date ranges of production of medium blue (1784-1859) transferprinted refined earthenware (Samford 2000:Table 5), and the fact that these transfer-printed sherds from the Browning site are whitewares, would seem to indicate that the occupation could have ranged from ca. 1830-1859. Central designs on transfer-printed refined earthenwares that featured Chinese elements were popular primarily before 1840 , while floral central designs (as well as border elements on plates) were popular "throughout the course of the 19th century (Samford 2000:73 and Figure 17). Peak periods of production of transfer-printed wares with floral designs was in the late 1840s.

Flown blue vessels became popular in the United States in the 1840s-1850s, especially those with landscape motifs (Samlord 2000:79 and Table 7). There are two sherds with blue sponged (or spattered, see Majewski and O'Brien 1987:161) decorations. This type of decorated whiteware was "produced in great quantities by British potteries throughout the nineteenth century, primarily for export, and in the United States after about 1850" (Majewski and O'Brien 1987:161).

Other ceramic sherds from the Browning site include several stoneware jug or crock sherds ( $n=4)$, yellowware sherds from bowls and crocks $(n=8)$, and sherds from glazed stoneware elbow pipes (Table 4). The stoneware sherds, from a minimum of two vessels, are from excavations only in the central part of the site, while the yellowware sherds (also from a minimum of two vessels, Figure 7, right) are distributed principally in the western part of the Browning site. The pipe sherds are also from excavations in the western part of the site (see Figure 1).

The stoneware sherds found in the archaeological deposits at the Browning site include salt-glazed sherds $(n=3)$, a brown-glazed ink bottle $(n=1)$ sherd, and yellowware $(n=8)$ (see Table 4$)$. These particular kinds of stoneware would have been manufactured and used between the 1830 s and ca. 1875. Salt glazing was one of the more commonly employed glazes in the manufacture of utilitarian stoneware (Greer 1981:180). The absence of salt-glazed stoneware sherds with a natural clay slipped interior surface suggests that these particular sherds are from stoneware vessels that were made before ca. 1870 (Lebo 1987:140).

The ink bottle basal sherd (see Figure 7, left) has a reddish-brown exterior glaze that extends near the base of the bottle, and it has a pinkish-paste. This particular stoneware vessel was probably made in England in the mid-19th century (ca. 1850s), would have had a paper label, and been stoppered with a cork (see Switzer 1974).

Yellowware began to produced in the $1820 \mathrm{~s}$ in England, but by the 1840 s it was also being manufactured in the United States, especially in the Midwest (Leibowitz 1985:4). The peak production of yellowware vessels was in the 1860s and 1870s, 


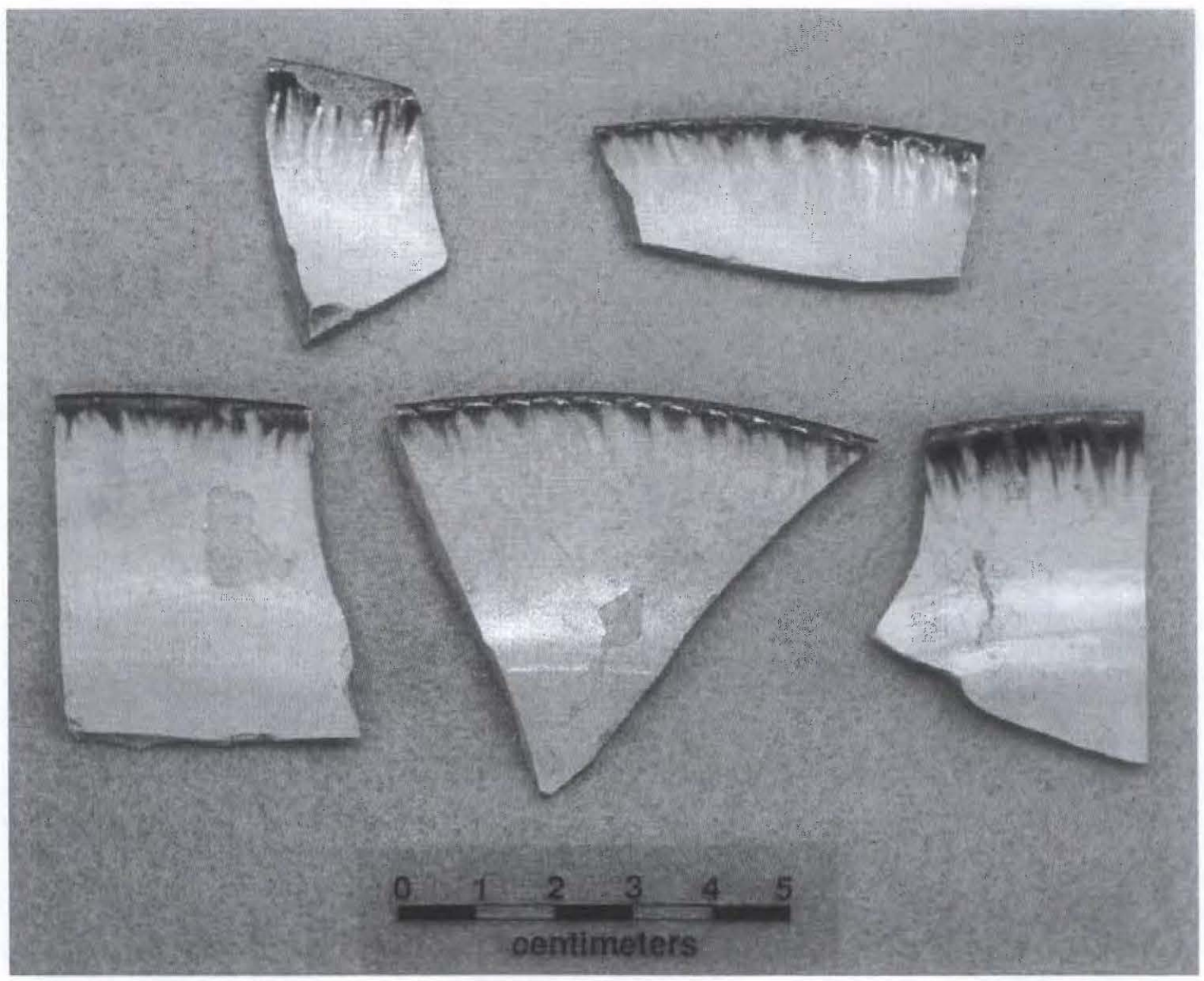

Figure 5. Blue Shell-edged plate or platter rims, non-scalloped, impressed lines. Provenience: top row, left to right: Surface; Unit 3, 0-10 cm; bottom row, left to right: Unit 40, 10-20 cm; Unit 42, 0-10 cm; Unit 19, 20-30 cm.

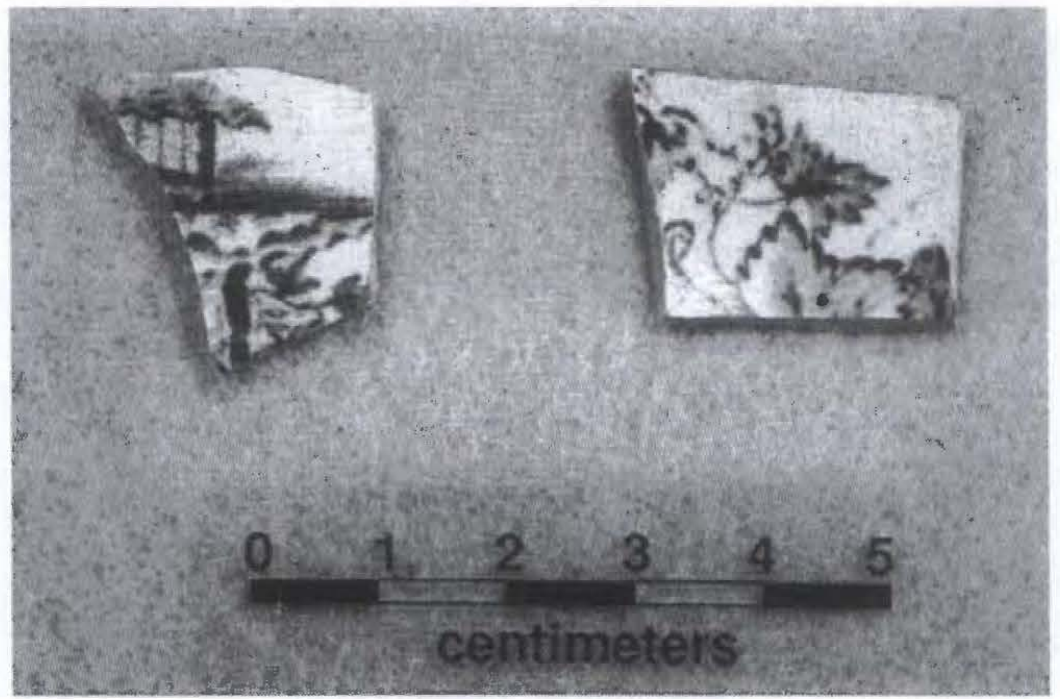

Figure 6. Blue transfer-printed body sherds. Provenience: left to right: Unit 17, $0-10 \mathrm{~cm}$; Unit $1,0-10 \mathrm{~cm}$. 
Table 4. Stoneware, Yellowware, and Ceramic Pipe Sherds from the Browning site.

\begin{tabular}{lccc}
\hline Provenience & Stoneware & Yellow ware & Stoneware Pipe \\
\hline ST $12,0-20$ & - & - & 1 (body) \\
ST $19,0-17$ & - & 1 & - \\
Unit $6,0-10$ & - & 1 & - \\
Unit $8,0-10$ & - & $1+$ & - \\
Unit $15,0-10$ & - & 1 & - \\
Unit $15,10-20$ & - & 1 & 1 (rim, elbow pipe) \\
Unit $16,10-20$ & - & 1 & - \\
Unit $24,0-10$ & - & 1 & - \\
Unit $29,10-20$ & 1 (ink bottle) & - & - \\
Unit 30, 20-30 & 1 (salt glazed) & - & - \\
Unit 31, 0-10 & - & 1 & - \\
Unit 32, 0-10 & 1 (salt glazed) & - & 2 \\
Unit 32, 20-30 & 1 (salt glazed) & - & - \\
\hline Totals & 4 & 8 & \\
\hline
\end{tabular}

+with blue and white bands

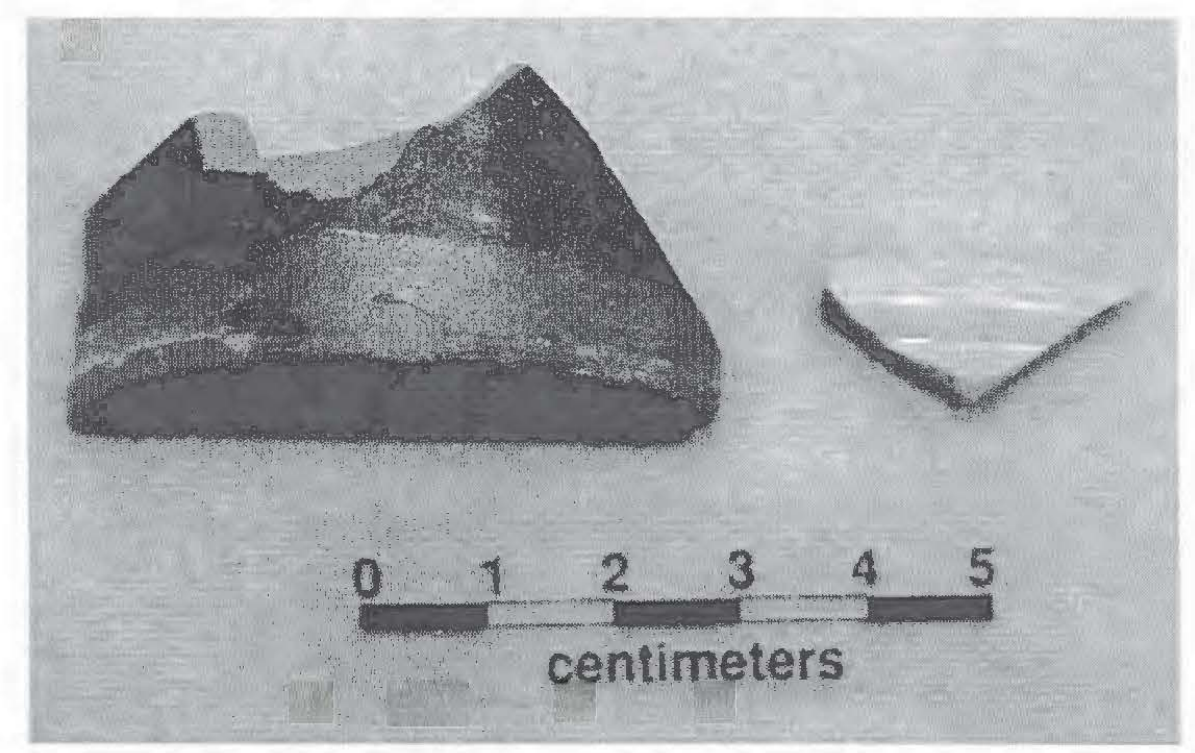

Figure 7. Stoneware sherd and yellowware sherd from the Browning site. Provenience: left to right: Unit $29,10-20 \mathrm{~cm}$; Unit $31,0-10 \mathrm{~cm}$.

although it was still being made in the early 1900s (Leibowitz 1985:14).

The pipe sherds from the Browning site are from early to mid-19th century stoneware forms. The elbow-shaped stoneware pipe is a reed stem pipe with a replaceable reed stem, and is moldmade, with a ribbed bowl. These sorts of pipes were made at several pottery kilns in the region, including the J. S. Nash factory in operation in Marion County, Texas, between 1850-1880 (Lebo 1988:282). Similar styles of molded elbow pipes have been recovered from 1837-1846 and 18521857 Anglo-American farmsteads in northeastern Texas as well as the $1840 \mathrm{~s}-1860$ s port of Monterey 
Table 5. Bottle Glass.

\begin{tabular}{lcc}
\hline $\begin{array}{l}\text { Provenience } \\
(\mathrm{cm} \text { bs) }\end{array}$ & $\begin{array}{c}\text { Aqua-colored } \\
\text { sherd }\end{array}$ & $\begin{array}{c}\text { Aqua-colored } \\
\text { medicine vial } \\
\text { sherd }\end{array}$ \\
\hline ST $4,0-20$ & 1 & - \\
ST 8, surface & 1 & - \\
Unit $6,10-20$ & 1 & - \\
Unit $13,20-30$ & 2 & - \\
Unit $14,10-20$ & - & - \\
Unit $15,0-10$ & 2 & - \\
Unit $15,10-20$ & 2 & - \\
Unit $23,30-40$ & 1 & 1 \\
Unit $34,20-30$ & 1 & - \\
Unit $41,0-10$ & 1 & - \\
\hline Totals & 12 & - \\
\hline
\end{tabular}

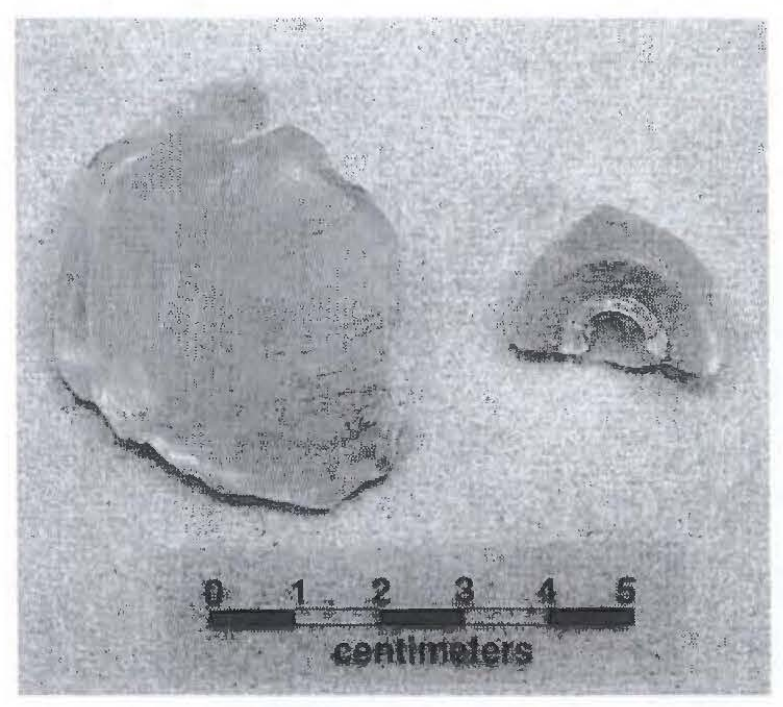

Figure 8. Aqua-colored bottle glass sherds. Provenience: left to right: Unit $41,0-10 \mathrm{~cm}$; Unit 14, 10-20 cm.

(Nelson and Pertula 2003; Perttula 1989:99; Perttula and Nelson 2010).

All of the bottle glass from the Browning site is aqua-colored (Table 5). One is from a small medicinal vial (Figure 8, right) with an iron-tipped pontil mark on its base (cf. Jones 2000:158-159), marking a method of holding the bottle during the finishing process that was used from the early 19 th century until the 1870s. These bottles appear to have been hand-blown and likely held medicines and liquor.
The principal metal artifacts (Tablc 6) from the Browning site are cut nails (1820-1891, see Wells 2000). Although they are not common, their occurrence suggests that the area of excavations at the site was in the vicinity of a wood-framed building, or a log structure (or in the vicinity of an area where structural/architectural artifacts were discarded), that may have had a mud cat chimney (see Jordan 1978). These nails occur in three small clusters in the western, central, and southern parts of the site (sce Figure 1).

Horse and stable artifacts include two horseshoe nails and an iron buckle (Figure 9, bottom row, left). There are also kitchen/domestic artifacts, such as an iron spoon handle (Figure 9, left), and a possible iron shaft/handle pull to a piece of wood furniture or a cabinet (Figure 9, bottom row, right). One plain 2-holed iron button is indicative of clothing/adornment (Figure 9, top row, left).

The two small and thin picces of tin (see Figure 9 , top row, right) may be from a cup, or various sorts of pans. Finally, there are several pieces of unidentifiable strips and fragments of iron that may be evidence of working/fabricating iron tools.

\section{CONCLUSIONS}

The Browning site has well-preserved archaeological cvidence of a 19 th century occupation along 
Table 6. Metal Artifacts.

\begin{tabular}{|c|c|c|c|c|c|c|c|}
\hline Provenience & Cut Nail & $\begin{array}{c}\text { Horseshoe } \\
\text { nail/horse } \\
\text { tackle/buckle }\end{array}$ & Utensils & $\begin{array}{l}\text { Iron strap } \\
\text { frag./UID } \\
\text { frag, }\end{array}$ & $\begin{array}{l}\text { Iron } \\
\text { button }\end{array}$ & $\begin{array}{l}\text { Tin } \\
\text { frag. }\end{array}$ & $\begin{array}{c}\text { Iron } \\
\text { shaft/ } \\
\text { handle } \\
\text { pull }\end{array}$ \\
\hline Unit $5,0-10$ & - & 1 (buckle) & - & - & - & - & - \\
\hline Unit $6,0-10$ & - & - & 1 (spoon) & - & - & - & - \\
\hline Unit 6, 20-30 & 1 & - & - & - & - & - & - \\
\hline Unit $\mathrm{I} 3,10-20$ & - & - & - & - & - & 1 & - \\
\hline Unit $13,20-30$ & 2 & - & - & - & - & - & - \\
\hline Unit $15,10-20$ & - & - & - & 1 & - & - & - \\
\hline Unit $17,0-10$ & 1 & - & - & - & - & - & - \\
\hline Unit $19,10-20$ & - & - & - & - & - & 1 & - \\
\hline Unit $20,10-20$ & - & - & - & 2 & - & - & - \\
\hline Unit $21,0-10$ & - & - & - & 2 & - & - & - \\
\hline Unit $22,10-20$ & - & - & - & - & 1 & - & - \\
\hline Unit $24,0-10$ & 1 & - & - & - & - & - & - \\
\hline Unit $24,10-20$ & - & - & - & 1 & - & - & - \\
\hline Unit $28,30-40$ & - & - & - & 1 & - & - & - \\
\hline Unit $30,10-20$ & 1 & - & - & - & - & - & - \\
\hline Unit $31,10-20$ & - & - & - & 2 & - & - & - \\
\hline Unit $33,20-30$ & 1 & - & - & - & - & - & - \\
\hline Unit $33,30-40$ & 1 & - & - & - & - & - & - \\
\hline Unit $34,20-30$ & 4 & - & - & 2 & - & - & - \\
\hline Unit $35,10-20$ & - & 1 (nail) & - & 1 & - & - & - \\
\hline Unit $36,10-20$ & 1 & - & - & - & - & - & - \\
\hline Unit $36,20-30$ & 1 & - & - & - & - & - & - \\
\hline Unit $37,10-20$ & 2 & - & - & 1 & - & - & - \\
\hline Unit $38,10-20$ & - & - & - & - & - & - & 1 \\
\hline Unit $38,20-30$ & - & 1 (nail) & - & - & - & - & - \\
\hline Unit $39,10-20$ & - & - & 1 & - & - & - & \\
\hline Unit $42,0-10$ & 1 & - & - & - & - & - & - \\
\hline Unit $43,20-30$ & - & - & - & 1 & - & - & - \\
\hline Totals & 17 & 3 & 1 & 15 & 1 & 2 & 1 \\
\hline
\end{tabular}

* This does not include a REM-UMC No. 12 NITRO CLUB shotgun shell from Unit 36 (20-30 cm)

a tributary to the Sabine River, in northern Smith County, Texas. Although no habitation leatures have been identified in the excavations conducted to date at the site, and the recovery of cut nails and an assortment of kitchen/domestic artifacts (refined earthenware plates and cups, stoneware vessel sherds, and bottle glass sherds) personal items (an iron button), and horse gear (horseshoe nails and an iron buckle), it seems clear that there was a structure built on the site that was lived in by at least one family, probably a farming family. The low density of artifacts across the site, as well as the relatively ephemeral nature of the archaeological deposits (ca. $20 \mathrm{~cm}$ thick, covering only ca. $500 \mathrm{~m}$, and little evidence of features such as foundations, pier stones, wells, or privies), suggest this probable farmstead may have been occupied for no more than a generation (ca. 20 years).

The best available evidence that speaks to the chronological age of the Browning site occupation is the decorated refined earthenware. The 


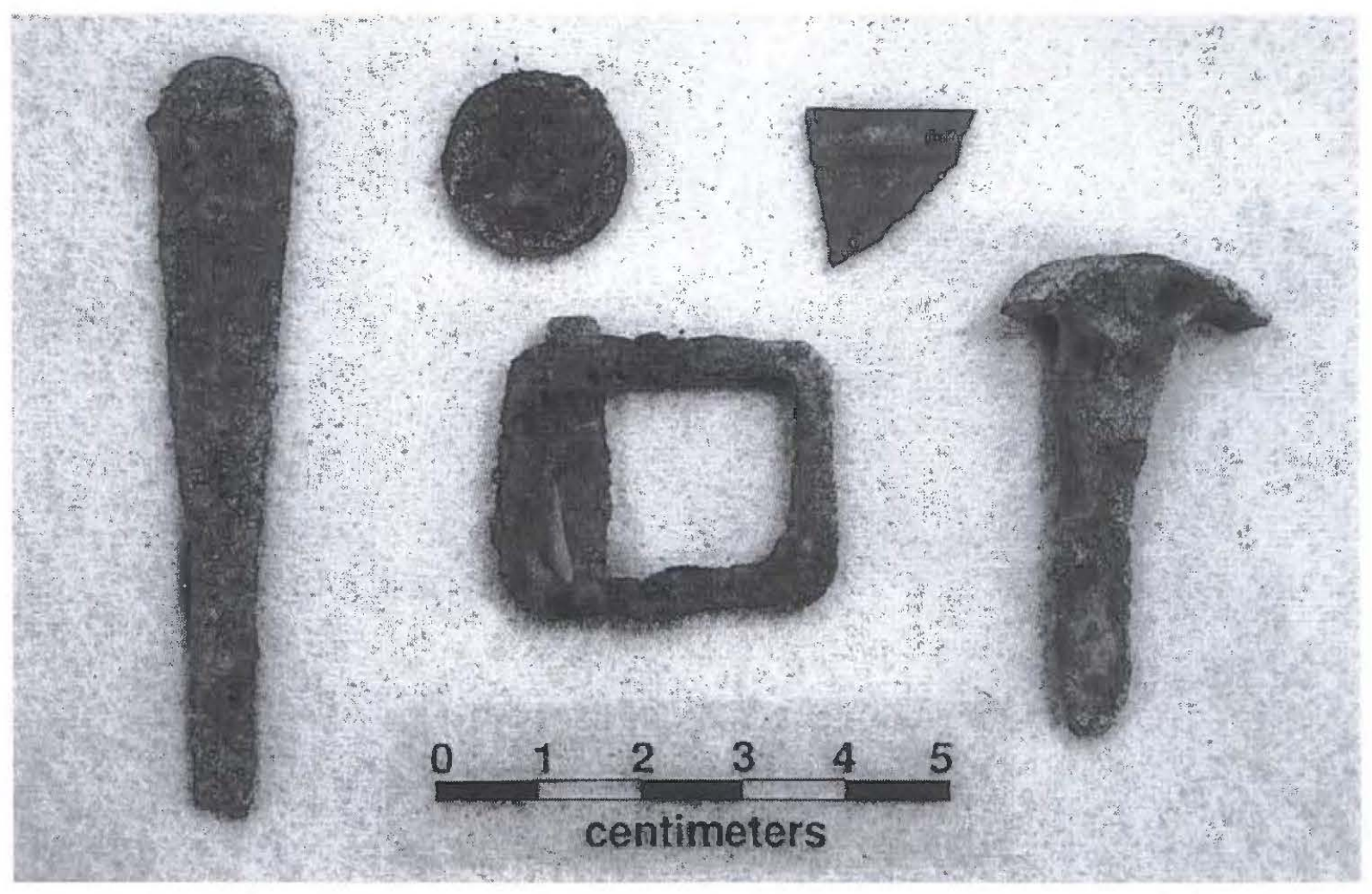

Figure 9. Metal artifacts from the Browning site: left: iron spoon handle; top row, left to right: 2-holed iron button; thin piece of tin (?); bottom row, left to right: metal buckle (horse tack); shaft/handle pull or large forged nail. Provenience: left, Unit 6, 0-10 cm; top row, left to right, Unit 22, 10-20 cm; Unit 13, 10-20 cm; bottom row, left to right: Unit 5, 0-10 $\mathrm{cm}$; Unit 38, 10-20 cm.

preponderance of the evidence (see Table 3 ) is consistent with a ca. 1840-1860 occupation, one that postdated the Cherokee occupation of East Texas. The historic occupation of the Browning site is most likely the product of an Anglo-American settlement in the Sabine River basin.

\section{REFERENCES CITED}

Diggs Jr., G. M., B. L. Lipscomb, M. D. Reedand R.J. O'Kennon 2006 Illustrated Flora of East Texas, Volume One: Introduction, Pteridophytes, Gymnosperms, and Monoconyledons Sida, Botanical Miscellany, No. 26. Botanical Research Institute of Texas, Fort Worth.

Greer, G. H.

1981 American Stoneware: The Art and Craft of Utilitarian Pottery. Schiffer Publishing Ltd., Exton, Pennsylvania.
Hatherly, D. T.

1993 Soil Survey of Smith County. United States Department of Agriculture, Soil Conservation Service, in cooperation with the Texas Agricultural Experiment Station and the Texas State Soil and Water Conservation Board.

Hunter, R. R. Jr. and G. L. Miller

1994 English Shell-edged Earthenware. Antiques CXLV (No. 3):432-443.

2009 Suitable for Framing: Decorated Shell-Edge Earthenware. Early American Life (August):8-19.

Jones, $\mathrm{O}$.

2000 Glass Bottle Push-Ups and Pontil Marks. In Approaches to Material Culture Research for Historical Archaeologists, compiled by D. R. Brauner. pp. 149-160. 2nd Edition. The Society for Historical Archaeology. California, Pennsylvania.

Jordan, T. G.

1978 Texas Log Buildings: A Folk Architecture. University of Texas Press, Austin. 
Lebo. S. A.

1987 Local Utilitarian Stonewares: A Diminishing Artifact Category. In Historic Buildings, Material Culture, and People of the Prairic Margin: Architecture. Artifacts, and Symthesis of Historic Archaeology: edited by D. H. Jurney and R. W. Moir, Pp. 121-142. Volume V. Archacology Research Program, Institute for the Study of Earth and Man, Southern Methodist University, Dallas.

1988 Local Utilitarian Stoneware Vessels: Developing a Regional Model of Stoneware Use on Farmsteads in Northeastem Texas during the Nineteenth and Early Twenticth Centuries. In Historic farming on the Hogwallow Prairie: Ethnoarchaeological Investigations of the Mountain Creek Area, North Central Texas, compiled by D. H. Jurney, S. A. Lebo, and M. M. Green, pp. 273-297. Joe Pool Lake Archaeological Project, Volume II. Archaeology Research Program, Institute for the Study of Earth and Man, Southern Methodist University, Dallas.

Leibowitı. J.

1985 Yellow Ware: The Transitional Ceramic. Schifler Publishing Ltd., West Chester, Pennsylvania.

Majewski, T. and M. J. O’Brien

1984 An Analysis of Historical Ceramics from the Central Salt River Valley' of Northeast Missouri. Report No. 3. American Archaeology Division. Department of Anthropology, University of Missouri. Columbia.

1987 The Use and Misuse of Nineteenth-Century English and American Ceramics in Archaeological Analysis. In Advances in Archaeological Method and Theon: Volume 11. edited by M. B. Schiffer, pp. 97-209. Academic Press, Inc., New York.

Nelson, B. and T, K. Perttula

2003 Archeological Survey along the Lake Bob Sandlin Shoreline, Camp, Franklin, and Tilus Counties, Texas. Report of Investigations No. 46. Archeological and Environmental Consultants, LLC. Austin.

Perttula, T. K. and B. Nelson

2010 Archaeological Investigations Alung James Bayou in Marion County. Texas and Caddo Parish. Louisiana. Journal of Northeast Texas Archaeology 32:1-50.

Perttula. T. K. (editor)

1989 The James Franks Site (41DT97): Excavations at a Mid-Nineteenth Century Farmstead in the South Sulphur River Valley, Cooper Lake Project, Texas. Contributions in Archaeology No. 7. Institute of Applied Sciences, University of North Texas, Denton.
Price. C. R.

1979 Nineteenth Cenury Ceramics in the Eastern Ozark Border Region: An Analysis of Refined Earthenware from Selected Sites in the ()zark Border Region of Southeast Missouri. Monograph Series No. 1. Center for Archacological Research, Southwest Missouri State University, Springfield.

Samford, P. M.

2000) Response to a Market: Dating Fnglish Underglaze Transfer-Printed Wares. In Approaches to Material Culture Research for Historical Archacologists. compiled by D. R. Brauner. pp. 56-85. 2nd Edition. The Society for Historical Archaeology, California. Pennsylvania.

Shafer, $\mathrm{H}$. and $\mathrm{M}$. Walters

2010 The Browning Site (4ISM195A) Lithics: Considering Patterns of Identity and Interaction through Lithic Analysis. Bulletin of the Texas Archeological Society 81:127-151.

Sussman, I.

2000 British Military Tableware, 1760-1830. In Approaches to Material Culture Research for Historic al Archaeologists, compiled by D. R. Brauner, pp. 44-55. 2nd Edition. The Society for Historical Archaeology. California. Pennsylvania.

Switzer, R. R.

1974 The Bertrand Bottles: A Study of 19th-Century Glass and Ceramic Containers. Publications in Archaeology No. 12. U.S. Department of the Interior. National Park Service. Washington. D.C.

Walters, $\mathrm{M}$.

2004a 41SM195^. The Browning Site. Journul of Northcast Texas Archaeology 20:1-42.

2004b 41SM195A. The Browning Site. Caddoun Archeo/ogy Journal 13 (3-4):19-20.

2009 Prehistoric Ceramics from the Browning Sitc (41SM195A). Journal of Northeast Texas Archaeology $29: 1-7$.

Wells, T.

2000) Nail Chronology: The Use of Technologically Derived Features. In Approaches to Material Culture Research for IIistorical Archaeologists, compiled by D. R. Brauner. pp. 318-339. 2nd Fdition. The Suciety for Historical Archaeology, Califomia, Pennsylvania. 